\title{
Independent perceptual learning in monocular and binocular motion systems
}

\author{
Zhong-Lin Lu*†, Wilson Chu*, Barbara Anne Dosher ${ }^{\ddagger}$, and Sophia Lee* \\ * Laboratory of Brain Processes, Departments of Psychology and Biomedical Engineering, and Neuroscience Graduate Program, University of Southern \\ California, Los Angeles, CA 90089; and ₹Department of Cognitive Sciences and Institute of Mathematical Behavioral Sciences, University of California, \\ Irvine, CA 92697
}

Communicated by Patrick Suppes, Stanford University, Stanford, CA, February 17, 2005 (received for review July 29, 2004)

Eye-transfer tests, external noise manipulations, and observer models were used to systematically characterize learning mechanisms in judging motion direction of moving objects in visual periphery (Experiment 1) and fovea (Experiment 2) and to investigate the degree of transfer of the learning mechanisms from trained to untrained eyes. Perceptual learning in one eye was measured over 10 practice sessions. Subsequent learning in the untrained eye was assessed in five transfer sessions. We characterized the magnitude of transfer of each learning mechanism to the untrained eye by separately analyzing the magnitude of subsequent learning in low and high external noise conditions. In both experiments, we found that learning in the trained eye reduced contrast thresholds uniformly across all of the external noise levels: $47 \pm 10 \%$ and $62 \pm 8 \%$ in experiments 1 and 2 , respectively. Two mechanisms, stimulus enhancement and template retuning, accounted for the observed performance improvements. The degree of transfer to the untrained eye depended on the amount of external noise added to the signal stimuli: In high external noise conditions, learning transferred completely to the untrained eye in both experiments. In low external noise conditions, there was only partial transfer of learning: $63 \%$ in experiment 1 and $54 \%$ in experiment 2 . The results suggest that template retuning, which is effective in high external noise conditions, is mostly binocular, whereas stimulus enhancement, which is effective in low external noise displays, is largely monocular. The two independent mechanisms underlie perceptual learning of motion direction identification in monocular and binocular motion systems.

interocular transfer | stimulus enhancement | external noise exclusion mechanisms of perceptual learning

erceptual learning, which involves improvements of human performance in perceptual tasks through training or practice, has been demonstrated in a large variety of perceptual tasks (1-10). Transfer of perceptual learning to modified forms of the same task or to different related tasks has been the primary behavioral tool for discovering what is learned and inferring the functional locus and physiological basis of that learning. Transfer has been evaluated in terms of retinal position $(1,7,11-13)$, eye of training $(1,3,12,14-16)$, orientation or spatial frequency $(1$, $7,15,17,18)$, and retinal size $(12,15,19)$, among other properties. In many cases, the observed pattern of transfer in combination with known properties of the visual system have led to conclusions about the neural mechanisms and locus of learning. In this study, we focused on transfer of perceptual learning in identifying motion direction of moving luminance modulations from trained to untrained eyes.

Previous studies on perceptual learning in motion-direction identification have found a large degree of transfer from trained to untrained eyes $(13,16,20, \S)$ and therefore suggested a largely binocular site of learning. This conclusion seems to be consistent with the binocularity of most middle temporal visual area neurons. However, it appears to be inconsistent with the functional architecture of human motion perception. Based on different temporal modulation transfer functions and pedestal immunities measured with monocular and interocular motion displays, Lu and Sperling $(21,22)$ proposed that luminance motion is computed by two monocular and one (less sensitive) binocular motion systems (23). A recent psychophysical study found that learning motion discrimination was possible when middle temporal visual area was presumably "knocked out" by using psychophysical procedures (24). Single-unit recording studies in monkeys also suggested that perceptual learning of motion direction could take place in sites located downstream from the superior temporal sulcus $(25, \pi)$.

There is one critical difference between the studies on the eye transfer of perceptual learning of motion-direction identification and the psychophysical studies on the monocular/binocular nature of motion systems: all of the studies on eye transfer of perceptual learning of motion direction used high-contrast motion displays with large amounts of random noise, whereas the studies on the monocular/binocular nature of motion systems used displays with signal contrasts near discrimination thresholds without added noise. It is possible that perceptual learning of motion-direction discrimination has a large monocular component in displays without added noise, although it is largely binocular in displays with large amounts of added noise.

Recent investigations of the mechanisms of perceptual learning based on the external noise method suggest that distinctive learning mechanisms are engaged in displays with and without added external noise (4, 19, 26-29). By using the perceptual template model (PTM) (30), perceptual inefficiencies are attributed to three limitations: internal additive noise that sets the absolute thresholds for perceptual tasks; perceptual templates, often not perfectly matched to the signal in the stimulus, allow unnecessary influence of external noise or distractors on performance; and internal multiplicative noise that increases with input stimulus energy diminishes the benefit from increasing stimulus contrast and therefore predicts Weber's law behavior. Three mechanisms of perceptual learning, each improving on one of the perceptual limitations, can be distinguished: "stimulus enhancement" reduces absolute thresholds by reducing internal additive noise; "perceptual template retuning" optimizes the perceptual template to exclude external noise or distractors; and "contrast-gain control reduction" decreases the impact of internal multiplicative noise. These three mechanisms exhibit signature performance patterns when the external noise method is used to measure and compare threshold vs. contrast (TvC) functions at during perceptual learning (27). Stimulus enhancement increases the relative gain (vs. internal additive noise) of both the signal and the external noise in the stimulus and improves performance only in low or zero external noise.

\footnotetext{
Abbreviations: PTM, perceptual template model; TvC, threshold vs. contrast. †To whom correspondence should be addressed. E-mail: zhonglin@usc.edu. §Griffiths, F. \& Chubb, C. (1995) Invest. Ophthalmol. Vis. Sci. 36, $\$ 377$ (abstr.). IZohary, E. \& Newsome, W. T. (1994) Invest. Ophthalmol. Vis. Sci. 35, 1663 (abstr.). () 2005 by The National Academy of Sciences of the USA
} 

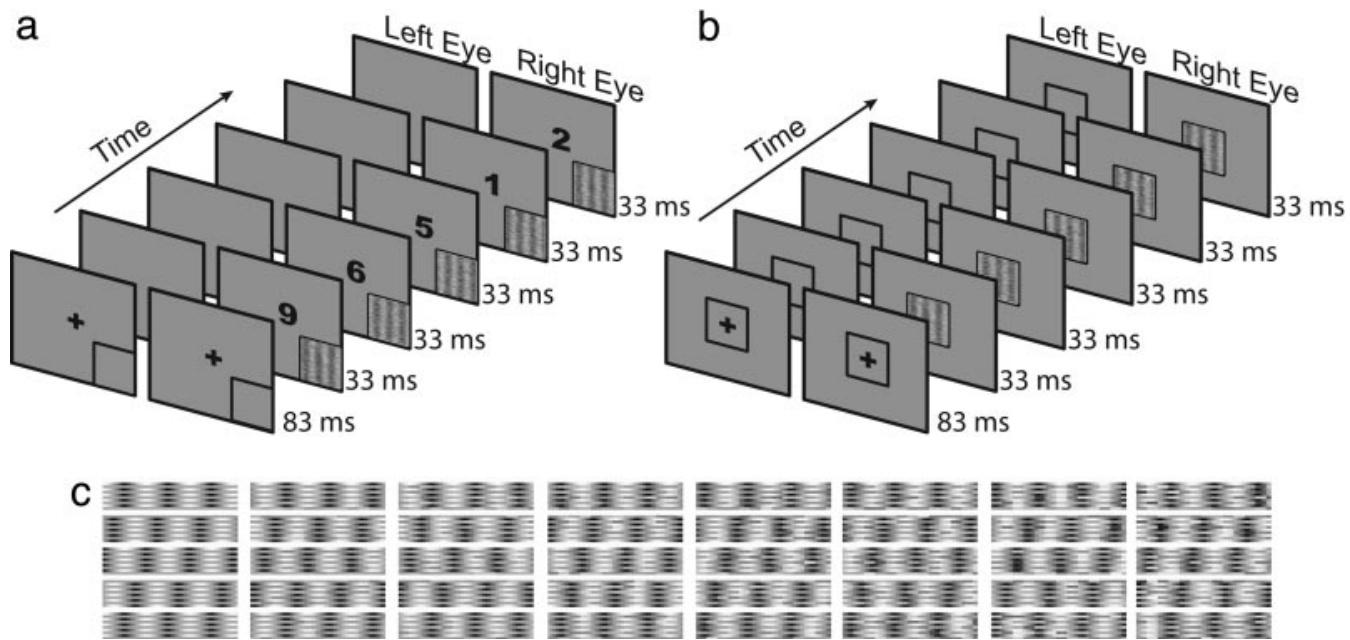

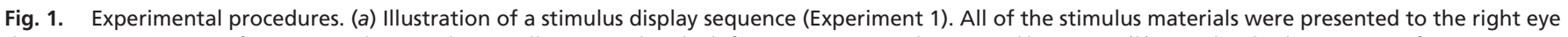

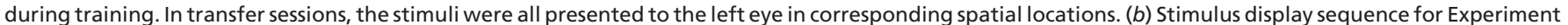

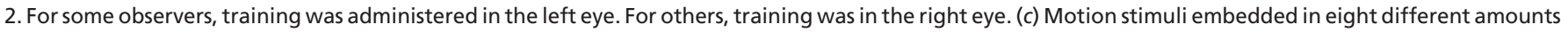
of external noise. Each image shows five frames of a rightward moving sine-wave grating. The phase shift between successive frames is $90^{\circ}$.

Perceptual template retuning improves the ability of the observer to exclude external noise and therefore improves performance only in high external noise. Contrast-gain control reduction increases system response to stimulus contrast yielding improvements throughout the full range of external noise.

The differential conditions of eye transfer in the prior motionlearning studies suggested that different learning mechanisms might have different degrees of eye transfer in motion-direction discrimination. In this study, we combined eye-transfer tests, the external noise method, and observer models to systematically investigate eye transfer of each learning mechanism in motiondirection identification in visual fovea and periphery. Observer performance in eight levels of external noise was measured in one eye in 10 training sessions and then in the other eye for another 5 transfer sessions. Learning curves and mechanisms of perceptual learning were characterized separately for the training and transfer sessions.

Most studies in the perceptual learning literature evaluate the magnitude of transfer with a three-step design, measuring prelearning performance in several conditions, training or practice in one particular condition, and measuring postlearning performance in all of the conditions. Transfer of perceptual learning is then evaluated by comparing postlearning and prelearning performance levels in each condition. Another design, frequently occurring in the cognitive learning literature but less often in perceptual learning, uses two steps: training or practice in one condition and further training or practice in other conditions. In this design, transfer of learning is evaluated by measuring the amount of further learning in the conditions not included in the training. Depending on the learning rate and the number of trials involved in obtaining reliable performance measures, the two designs have different pros and cons (31). Because measurements of TvC functions at two performance levels requires 1,000 trials or so, it is impractical to use the first design to obtain initial performance levels in both eyes without inducing significant learning in them. We instead chose the second design in this study. Here, the magnitude and mechanisms of subsequent learning in transfer sessions were used to characterize the degree of transfer of each learning mechanism. If a learning mechanism is substantially trained and then transferred completely, no subsequent learning would be associated with this mechanism; otherwise, the mechanism would exhibit subsequent learning. We quantified the magnitude of transfer with

$$
\text { transfer index }=1-\frac{\text { total_threshold_reduction(transfer) }}{\text { total_threshold_reduction(training) }} \text {. }
$$

Ranging from 0 to 1 , the index is 0 in the absence of any transfer and 1 in complete transfer.

\section{Materials and Methods}

Experiment 1. Observers identified the motion direction of a moving luminance sine-wave grating embedded in white noise in the visual periphery while performing a central fixation task (Fig. 1a). The central fixation task was used to encourage observers to maintain fixation. All of the stimulus materials were presented to one eye ("the trained eye") in 10 training sessions and then to the other "untrained eye" in 5 transfer sessions. A blank screen with background luminance was shown to the eye that did not receive stimulus materials.

The stimuli were displayed on a Eizo Nanao Technology Flexscan 6600 monitor (Torrance, CA) with a P4 phosphor, a refresh rate of $120 \mathrm{~Hz}$, and a background luminance of $27 \mathrm{~cd} / \mathrm{m}^{2}$. Each trial started with a 500-ms fixation display. A string of five characters, containing either a " 5 " or an " $\mathrm{S}$ " in the third temporal position and two randomly selected digits other than 5, replaced the fixation cross in the center of the display. Each character was shown for $33.3 \mathrm{~ms}$. Simultaneous with the onset of the character string, five frames of a moving sine-wave grating (spatial frequency, 2.03 cycles per degree; temporal frequency, $7.5 \mathrm{~Hz}$; size, $1.54^{\circ} \times 1.54^{\circ}$; center displaced $3.2^{\circ}$ horizontally and $2.4^{\circ}$ vertically from the fixation) embedded in external noise appeared in the stimulus quadrant, each lasting $33.3 \mathrm{~ms}$. The sine-wave grating, with a random initial phase, moved either to the left or right, with $90^{\circ}$ phase shifts between successive frames. External noise images were constructed from $0.03 \times 0.12$-pixel patches with identically distributed contrasts drawn independently from Gaussian distributions with mean of 0 and standard deviation (SD) of $N_{\text {ext }} \in\{0,0.02,0.04,0.08,0.12,0.16,0.25,0.33\}$. The maximum SD was set at 0.33 to conform reasonably well with Gaussian distributions. External noise was added to the signal sine-wave gratings by means of spatial and temporal integration: In a given frame, signal and external noise were 

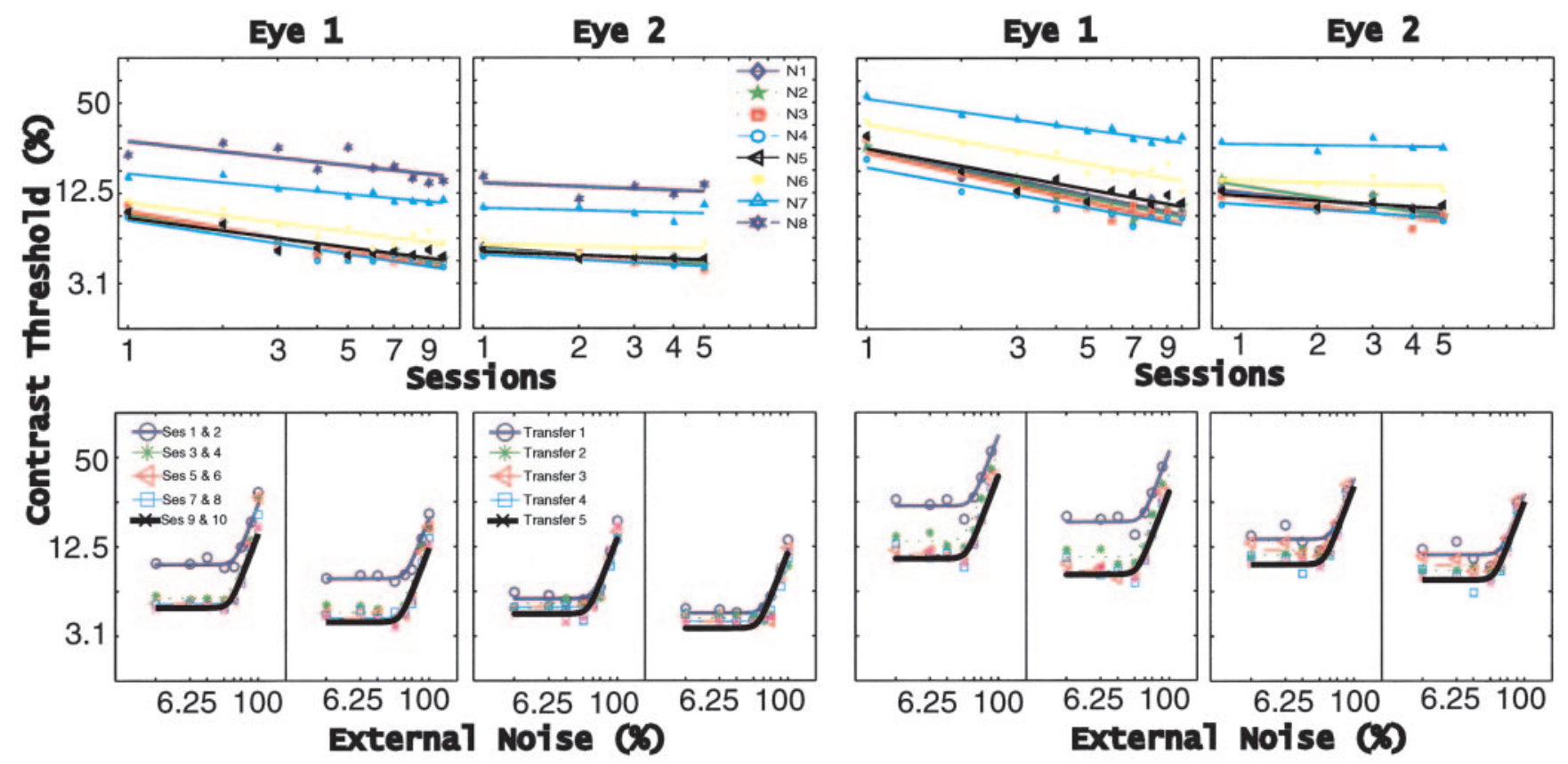

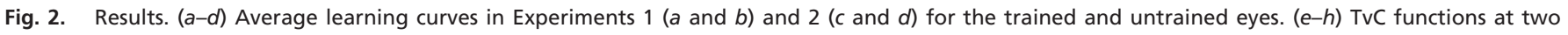
performance criterion levels [79.3\% (Left) and 70.7\% (Right) correct] in Experiments 1 (e and $f$ ) and 2 ( $g$ and $h$ ) in the trained and untrained eyes.

displayed in alternative $0.03^{\circ}$ rows; across frames, the pixels in a given row were alternately drawn from signal and noise images (Fig. 1c).

Observers identified the character ( 5 or $S$ ) in the central task and the direction of motion in the periphery. An auditory beep followed each correct response. Observer's threshold contrasts at two performance criterion levels were estimated for the peripheral task at each of eight external noise levels by using two interleaved staircase procedures (32). The two staircases estimated thresholds at $79.3 \%$ and $70.7 \%$ correct, corresponding to $d^{\prime}$ of 1.634 and 1.089 in two alternative forced-choice identification, by increasing contrast by $10 \%\left(C_{\text {new }}=1.1 C\right)$ after each error and reducing contrast by $10 \%\left(C_{\text {new }}=0.9 C\right)$ after three or two correct responses, respectively. Measurements of contrast thresholds at two criterion levels are critical in discriminating mixtures of perceptual learning mechanisms (27).

Three observers, Q.K., R.K., and S.L., with corrected-tonormal vision and naïve to the purpose of the experiment, participated in the study. The right eye is the dominant eye for R.K. and S.L., and the eye dominance of Q.K. is unknown. They each ran 10 training and then 5 transfer sessions. There were 1,120 trials per session, 80 and 60 trials per 3-down-1-up and 2 -down-1-up staircases. Each session lasted $\approx 45 \mathrm{~min}$. All in all, each observer ran 16,800 trials.

Experiment 2. The method for Experiment 2 was identical to that of Experiment 1 except that the motion stimuli were presented in fovea, there was no fixation task (Fig. 1b), and the initial training eye was different for different subjects. Three observers, T.J., U.P., and Y.Y., all with normal or corrected-to-normal vision and naïve to the purpose of the experiment, participated in the study. For T.J. (dominant eye, right), the initial training was performed in the right eye. For U.P. (dominant eye, right) and Y.Y. (dominant eye, right), the initial training was performed in the left eye.

\section{Results}

Experiment 1. The central task was used to encourage observers to maintain fixation. Central task performance was essentially constant, and good, across all training and transfer sessions as follows: $83 \pm 9 \%, 87 \pm 3 \%$, and $83 \pm 4 \%$ for the three observers. External noise level in the perceptual task did not significantly affect accuracy on the central task $(P>0.10)$.

An average learning curve (log threshold contrast as a function of log training days) for each external noise condition was calculated for training and transfer sessions by averaging threshold contrasts across observers and performance criteria for separate training days. The learning curves are shown in Fig. 2 $a$ and $b$ for the training and transfer phases of the experiment. Learning rates were estimated by using log-log linear regressions of the learning curves, consistent with power-law learning (33-35).

Over the 10 initial training sessions, practice reduced thresholds in the trained eye in all of the external noise conditions $(P<$ $0.015)$ with an average of $47 \pm 10 \%$ total reduction and a rate of $-0.29 \pm 0.06 \mathrm{log}$ units reduction per log unit of training session. The existence and magnitude of subsequent learning in the untrained eye depended on the external noise condition. Subsequent learning was significant in three $(P<0.03)$ and marginally significant in two $(P<0.09)$ of the five lowest external noise conditions and not significant in the highest three external noise conditions $(P>0.50)$. In the lowest five external noise conditions, contrast threshold reduced on average a total of $20 \pm$ $5 \%$ at a rate of $-0.12 \pm 0.03 \log$ units of reduction per log session. In the highest three external noise conditions, threshold reduced $4 \pm 8 \%$ on average over five transfer sessions at a rate of $-0.06 \pm 0.02 \log$ units per log session.

The transfer index (Eq. 1) was calculated for the low and high external noise conditions separately and is shown in Fig. 3. In the lowest five external noise conditions, the average transfer index was $55 \pm 12 \%$, significantly less than $1.0(P<0.05)$. In the highest three external noise conditions, the average transfer index was $89 \pm 17 \%$, not significantly different from $1.0(P>$ $0.25)$. The results suggest that training in one eye transferred completely to the other eye in high external noise conditions. There was only partial transfer of learning from the trained to the untrained eyes in low external noise conditions.

Thresholds, averaged over observers, are shown as functions 


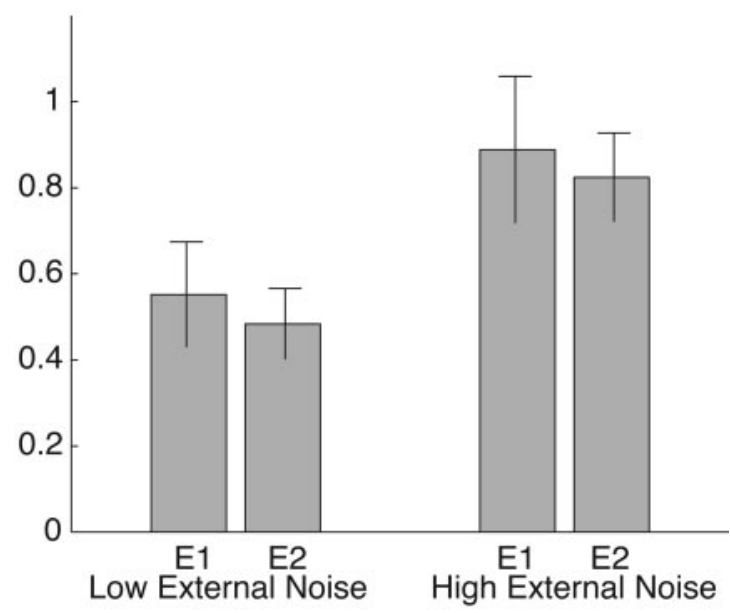

Fig. 3. Transfer indices in Experiment 1 (E1) and Experiment 2 (E2) in low and high external noise conditions.

of external noise contrast (TvC functions) at the two performance criterion levels in Fig. $2 e$ and $f$. The TvC functions were pooled over every two training sessions (Left) and displayed for each single transfer session (Right). The ratio of thresholds corresponding to $79.3 \%$ and $70.7 \%$ correct is essentially constant across the eight noise levels and practice in the training (mean, 1.26; SD, 0.15) and transfer sessions (mean, 1.26; SD, 0.16). The observed ratio constancy across external noise and practice levels indicates that practice did not alter contrast-gain control properties of the perceptual system $(27,30)$.

TvC functions over training days were fit with the PTM to characterize the mechanisms of learning (see Appendix). The data for the two eyes were fit separately. In the trained eye, performance improved by means of a mixture of stimulus enhancement and template retuning, estimated by the PTM as an $87 \%$ internal additive noise reduction (or an equivalent $658 \%$ stimulus enhancement) and a $38 \%$ external noise exclusion (by means of template retuning) across the training sessions. The best-fitting model, accounting for $98.5 \%$ of the variance, is statistically equivalent to the most saturated model that assumes all three mechanisms of perceptual learning $(P>0.90)$ and is superior to all its subset models $(P<0.00001)$.

In the untrained eye, subsequent learning during transfer sessions was explained by learning-induced stimulus enhancement in low external noise $(51 \%$ internal additive noise reduction or, equivalently, $103 \%$ stimulus enhancement) with smaller $(7 \%)$ although significant template retuning in high external noise. The best fitting model $\left(r^{2}=98.9 \%\right)$ is statistically equivalent to the most saturated model $(P>0.90)$ and is superior to all its subset models $(P<0.01)$.

The learning parameters are listed in Table 1 for individual observers and their average. The pattern of results is consistent across the observers.

Experiment 2. The learning curves for Experiment 2 are shown in Fig. $2 c$ and $d$. Over the 10 initial training sessions, practice reduced thresholds in the trained eye in all of the external noise conditions $(P<0.001)$ with on average a $62 \pm 8 \%$ total reduction and a rate of $-0.40 \pm 0.06 \log$ units reduction per log unit of training. Similar to Experiment 1, the existence and magnitude of subsequent learning in the untrained eye depended on the

"Because Y.Y. could not perform the task in the highest external noise condition, all average data only included seven noise levels. Data from all eight noise levels were included in analyzing the data of T.J. and U.P. external noise condition. Subsequent learning in the untrained eye over the five transfer sessions was significant in two $(P<$ $0.03)$ and marginally significant in three $(P<0.10)$ of the five lowest external noise conditions and not significant in the highest two external noise conditions $(P>0.35)$. In the lowest five external noise conditions, contrast threshold reduced on average a total of $30 \pm 10 \%$ at a rate of $-0.22 \pm 0.08 \log$ units of reduction per log session. In the highest two external noise conditions, there was on average a total of $11 \pm 5 \%$ threshold reduction over five transfer sessions at an average rate of $-0.05 \pm 0.03 \log$ units per $\log$ session.

The transfer index (Eq. 1) was calculated for the low and high external noise conditions separately (Fig. 3). In the lowest five external noise conditions, the average transfer index was $48 \pm$ $8 \%$, significantly less than $1.0(P<0.02)$. In the highest three external noise conditions, the average transfer index was $83 \pm$ $10 \%$, not significantly different from $1.0(P>0.10)$. The results suggest that training in one eye transferred almost completely to the other eye in high external noise conditions. There was only partial transfer of learning from the trained to the untrained eyes in low external noise conditions.

The average TvC functions are shown in Fig. $2 g$ and $h$ for the two criterion levels. The ratio of thresholds corresponding to $79.3 \%$ and $70.7 \%$ correct was essentially constant across the eight noise levels and practice in the training (mean, 1.28; SD, 0.03 ) and transfer sessions (mean, 1.27; SD, 0.03), indicating that practice did not alter contrast-gain control properties of the perceptual system.

The TvC functions for the two eyes were fit with the PTM separately. In the trained eye, performance improved by means of a mixture of stimulus enhancement $(91.4 \%$ internal additive noise reduction) and external noise exclusion (46.7\%) across the training sessions. The best fitting model $\left(r^{2}=97.7 \%\right)$ is statistically equivalent to the most saturated model that assumes all three mechanisms of perceptual learning $(P>0.90)$ and is superior to all its subset models $(P<0.00001)$.

In the untrained eye, subsequent learning during transfer sessions was explained by learning-induced stimulus enhancement (69.8\% internal additive noise reduction or, equivalently, $231 \%$ stimulus enhancement) only across transfer sessions. The best fitting model $\left(r^{2}=97.6 \%\right)$ is statistically equivalent to the most saturated model $(P>0.50)$ and is superior to all its subset models $(P<0.00001)$. The pattern of results is consistent across the observers (Table 1$)$.

\section{Summary and Discussion}

In two experiments, one conducted in visual periphery and the other in fovea, we found that monocular learning improved performance (reduced contrast thresholds) with virtually equal (log) magnitude across a wide range of external noise levels with no significant change in central task performance. We identified a mixture of stimulus enhancement in clear or low noise displays and template retuning in high external conditions as the mechanisms of perceptual learning. The degree of transfer of learning from the trained to the untrained eyes depended on the external noise level. In high external noise conditions, learning transferred (nearly) completely to the untrained eye, and training in the second eye yielded almost no (Experiment 1) or no (Experiment 2) further improvement. In low external noise conditions, only $63 \%$ (Experiment 1) and 54\% (Experiment 2) of transfer was found. Performance further improved by means of stimulus enhancement in the untrained eye during the five transfer sessions.

In displays with no or little added external noise, observer performance is limited by internal noise and can only be improved by stimulus enhancement. The improvements in the trained eye only transferred partially to the untrained eye. Subsequent learning by means of stimulus enhancement in the 
Table 1. Summary of learning parameters

\begin{tabular}{|c|c|c|c|c|c|c|c|c|}
\hline \multirow[b]{2}{*}{ Parameter } & \multicolumn{4}{|c|}{ Experiment 1} & \multicolumn{4}{|c|}{ Experiment 2} \\
\hline & QK & RK & SL & Avg & TJ & UP & YY & Avg \\
\hline \multicolumn{9}{|c|}{ Training } \\
\hline$A_{\text {add }}(2)$ & 0.039 & 0.427 & 0.381 & 0.208 & 0.117 & 0.120 & 0.211 & 0.195 \\
\hline$A_{\text {add }}(3)$ & 0.022 & 0.600 & 0.219 & 0.158 & 0.053 & 0.051 & 0.142 & 0.103 \\
\hline$A_{\text {add }}(4)$ & 0.017 & 0.522 & 0.297 & 0.161 & 0.026 & 0.052 & 0.231 & 0.099 \\
\hline$A_{\text {add }}(5)$ & 0.017 & 0.248 & 0.305 & 0.132 & 0.080 & 0.019 & 0.149 & 0.086 \\
\hline$A_{\text {ext }}(2)$ & 0.714 & 0.731 & 0.856 & 0.749 & 0.688 & 0.800 & 0.738 & 0.700 \\
\hline$A_{\text {ext }}(3)$ & 0.700 & 0.700 & 0.782 & 0.707 & 0.585 & 0.695 & 0.566 & 0.580 \\
\hline$A_{\text {ext }}(4)$ & 0.643 & 0.522 & 0.686 & 0.643 & 0.569 & 0.730 & 0.491 & 0.530 \\
\hline$A_{\text {ext }}(5)$ & 0.632 & 0.583 & 0.663 & 0.625 & 0.644 & 0.700 & 0.478 & 0.533 \\
\hline$r^{2}$ & 0.969 & 0.963 & 0.990 & 0.983 & 0.975 & 0.980 & 0.925 & 0.977 \\
\hline \multicolumn{9}{|c|}{ Transfer } \\
\hline$A_{\text {add }}(2)$ & 0.552 & 1.25 & 0.478 & 0.739 & 0.510 & 0.448 & 0.515 & 0.471 \\
\hline$A_{\text {add }}(3)$ & 0.795 & 0.885 & 0.400 & 0.576 & 0.967 & 0.403 & 0.556 & 0.609 \\
\hline$A_{\text {add }}(4)$ & 0.636 & 1.05 & 0.356 & 0.591 & 0.495 & 0.203 & 0.301 & 0.307 \\
\hline$A_{\text {add }}(5)$ & 0.619 & 0.498 & 0.426 & 0.391 & 0.413 & 0.280 & 0.255 & 0.302 \\
\hline$A_{\text {ext }}(2)$ & 0.736 & 1.0 & 0.847 & 0.829 & 0.843 & 1.0 & 1.0 & 1.0 \\
\hline$A_{\text {ext }}(3)$ & 0.878 & 1.0 & 0.785 & 0.859 & 0.953 & 1.0 & 1.0 & 1.0 \\
\hline$A_{\text {ext }}(4)$ & 0.762 & 1.0 & 0.784 & 0.807 & 0.755 & 1.0 & 1.0 & 1.0 \\
\hline$A_{\text {ext }}(5)$ & 0.972 & 1.0 & 0.753 & 0.930 & 0.877 & 1.0 & 1.0 & 1.0 \\
\hline$r^{2}$ & 0.974 & 0.970 & 0.985 & 0.990 & 0.977 & 0.970 & 0.908 & 0.976 \\
\hline
\end{tabular}

Avg, average.

untrained eye reduced the contrast thresholds to comparable levels of the trained eye in the end of training. The results suggest that stimulus enhancement has a large monocular component. In high external noise conditions, observer performance is limited by external noise and can only be improved by template retuning. The effects of template retuning in the trained eye transferred virtually completely to the untrained eye. The transfer results in high external noise conditions are completely consistent with those of previous studies on eye-specificity of perceptual learning of motion direction discrimination in noisy displays $(13,16$, 20). The observed partial eye-transfer of perceptual learning in low external noise is consistent with and provides further support for the existence of monocular luminance motion systems (2123). In a related study, we (Z.-L.L., W.C., B.A.D., and S.L., unpublished data) investigated transfer of perceptual learning of Gabor orientation identification across eyes by using the same design as in Experiment 1. We found complete transfer of perceptual learning from trained to untrained eyes across all of the noise levels. The particular pattern of transfer observed in the current study is probably unique for motion perception.

According to Lu and Sperling (22), there are two monocular and one (less sensitive) binocular luminance motion systems. All three systems provide inputs to motion integration and decision on motion direction. One possible interpretation of the relative sensitivities of the monocular and binocular motion systems is that the monocular systems have less internal noise and the binocular system is limited by a higher amount of internal noise. Training in one eye affects both the corresponding monocular motion system and the binocular motion system. In displays with no or little added external noise, both the monocular and the binocular motion systems are limited by internal noise; effective learning should mostly result from further reduction of the internal noise in the more sensitive monocular motion system. Because the relative sensitivity of the monocular and binocular system is $\approx 2: 1(22)$, not infinity, a certain amount of training of the binocular system is of course inevitable. This characteristic could account for the observed partial transfer of learning from the trained to the untrained eyes. In high external noise displays, the monocular and binocular motion systems are mostly limited by external rather than internal noise; learning by means of template retuning improves both systems. Our results suggest that the binocular system might be more susceptible to template retuning, consistent with recent neurophysiological results in largely binocular middle temporal visual area neurons (36).

Perceptual learning reflects plasticity in the adult brain. The physiological basis of the plasticity underlying perceptual learning is a current topic of research. Although topographical reorganization of cortical maps as a result of perceptual learning has been documented in primary somatosensory cortex $(37,38)$ and primary auditory cortex (39-42), comparisons of the primary visual cortices before and after perceptual learning have not found any significant topographical map reorganization (43-45). Although one study (44) found some modest changes of orientation tuning in V1 that accounted for a small fraction of the behavioral improvement, others $(43,45)$ failed to find any pronounced changes in neural responsitivity associated with behavioral improvements with tasks suited for early visual cortical areas. A recent computational model of perceptual learning** accounted for a very complex behavioral data set in a nonstationary environment through incremental channel reweighting without altering early stages of visual processing, lending an existence proof of reweighting of early visual channels as a plausible mechanism of perceptual learning $(26,45,46)$.

The results from this study suggest that perceptual learning in identifying motion direction of moving luminance-defined objects involves two independent mechanisms. The existence of the monocular stimulus enhancement learning mechanism in displays with no or little added external noise does not necessarily imply, however, that the learning mechanism resides in monocular neurons. Even though binocular neurons receive inputs from both eyes, the internal noise in the two monocular inputs to them might be uncorrelated. Performance improvements of binocular neurons may reflect reduction of internal noise associated with each monocular input and therefore is eye specific (47). A combined taxonomy of mechanisms and physiological

**Petrov, A., Dosher, B. A. \& Lu, Z.-L. (2003) J. Vision 3, p. 670a, abstr. 670. 
sites of perceptual learning is necessary for the understanding of the nature of perceptual learning.

\section{Appendix}

The PTM (30) is an observer model that quantitatively models human performance in signal detection and discrimination. A PTM consists of the following five components: $(i)$ a perceptual template; (ii) a nonlinear transducer function, $\|\cdot\| \gamma$; (iii) a multiplicative Gaussian internal noise whose SD is proportional (with a factor of $N_{\text {mul }}$ ) to the total energy in the stimulus after the nonlinear transformation; (iv) an additive internal noise whose amplitude $\left(N_{\text {add }}\right)$ is independent of the stimulus strength; and $(v)$ a decision process (see ref. 30 for the formal development and quantitative tests for the form of the PTM).

The three mechanisms of perceptual learning in PTM, stimulus enhancement, external noise exclusion, and multiplicative noise reduction, provide a complete mathematical basis to accommodate all possible systematic patterns of performance improvements. The mechanisms of perceptual learning are modeled by multiplying the corresponding noise in the PTM with learning parameters $A_{\text {add }}(t), A_{\text {ext }}(t)$, and $A_{\text {mul }}(t)$ in each training block $t(19,26,27)$. In the most saturated PTM that includes all

1. Karni, A. \& Sagi, D. (1991) Proc. Natl. Acad. Sci. USA 88, 4966-4970.

2. Fiorentini, A. \& Berardi, N. (1980) Nature 287, 43-44.

3. Fahle, M. \& Edelman, S. (1993) Vision Res. 33, 397-412.

4. Saarinen, J. \& Levi, D. M. (1995) Vision Res. 35, 519-527.

5. McKee, S. P. \& Westheimer, G. (1978) Percept. Psychophys. 24, 258-262.

6. Ahissar, M. \& Hochstein, S. (1997) Nature 387, 401-406.

7. Shiu, L.-p. \& Pashler, H. (1992) Percept. Psychophys. 52, 582-588.

8. Ball, K. \& Sekuler, R. (1982) Science 218, 697-698.

9. Ramachandran, V. S. \& Braddick, O. (1973) Perception 2, 371-376.

10. Watanabe, T., Nanez, J. E. S., Koyama, S., Mukai, I., Liederman, J. \& Sasaki, Y. (2002) Nature Neurosci. 5, 1003-1009.

11. Berardi, N. \& Fiorentini, A. (1987) J. Physiol. 384, 633-647.

12. Ahissar, M. \& Hochstein, S. (1996) Vision Res. 36, 3487-3500.

13. Schoups, A. A., Vogels, R. \& Orban, G. A. (1995) J. Physiol. 483, 797-810.

14. Schoups, A. A. \& Orban, G. A. (1996) Proc. Natl. Acad. Sci. USA 93, $7358-7362$.

15. Fiorentini, A. \& Berardi, N. (1981) Vision Res. 21, 1149-1158.

16. Ball, K. \& Sekuler, R. (1987) Vision Res. 27, 953-965.

17. Liu, Z. \& Weinshall, D. (2000) Vision Res. 40, 97-109.

18. Liu, Z. \& Vaina, L. M. (1998) Cog. Brain Res. 6, 347-349.

19. Lu, Z.-L. \& Dosher, B. A. (2004) J. Vision 4, 44-56.

20. Vaina, L. M., Sundareswaran, V. \& Harris, J. G. (1995) Cog. Brain Res. 2, $155-163$.

21. Lu, Z.-L. \& Sperling, G. (1995) Vision Res. 35, 2697-2722.

22. Lu, Z.-L. \& Sperling, G. (2001) J. Opt. Soc. Am. A 18, 2331-2370.

23. Carney, T. (1997) Vision Res. 37, 2361-2368.

24. Lu, H., Qian, N. \& Liu, Z. (2004) Vision Res. 44, 1817-1825.

25. Zohary, E., Celebrini, S., Britten, K. H. \& Newsome, W. T. (1994) Science 263, 1289-1292.

26. Dosher, B. A. \& Lu, Z.-L. (1998) Proc. Natl. Acad. Sci. USA 95, 13988-13993.

27. Dosher, B. A. \& Lu, Z.-L. (1999) Vision Res. 39, 3197-3221.

28. Gold, J., Bennett, P. J. \& Sekuler, A. B. (1999) Nature 402, 176-178. three mechanisms of perceptual learning, thresholds are expressed as functions of external noise by in the following equation:

$c_{\tau}=\frac{1}{\beta}\left[\frac{\left(1+\left(A_{\mathrm{mul}}(t) N_{\mathrm{mul}}\right)^{2}\right)\left(A_{\mathrm{ext}}(t) N_{\mathrm{ext}}\right)^{2 \gamma}+\left(A_{\mathrm{add}}(t) N_{\mathrm{add}}\right)^{2}}{\left(1 / d^{\prime 2}-\left(A_{\mathrm{mul}}(t) N_{\mathrm{mul}}\right)^{2}\right)}\right]^{\frac{1}{2 \gamma}}$.

To identify the mechanism(s) of perceptual learning, all eight possible versions of PTMs, consisting of various combinations of the three mechanisms of perceptual learning, were fit to each set of $\mathrm{TvC}$ functions by using a least-square minimization procedure. The results were compared statistically by using nested model tests based on F-statistics (48). The best fitting model, statistically equivalent to the fullest yet with the minimum number of parameters, identified the mechanism(s) of perceptual learning.

This work was supported by National Science Foundation Grants BCS-9911801 and BCS-9910678, National Institute of Mental Health Grant 1 R01 MH61834-01, and Air Force Office of Scientific Research Grant F49620-01-1-0109.

29. Li, R. W., Levi, D. M. \& Klein, S. A. (2003) Nature Neurosci. 7, 178-183. 30. Lu, Z.-L. \& Dosher, B. A. (1999) J. Opt. Soc. Am. A 16, 764-778.

31. Pennington, N. \& Rehder, B. (1995) in The Psychology of Learning and Motivation: Advances in Research and Theory, ed. Medin, D. L. (Academic, San Diego), Vol. 33, pp. 223-289.

32. Levitt, H. (1971) J. Acoust. Soc. Am. A 49, 467-477.

33. Anderson, J. R. \& Fincham, J. M. (1994) J. Exp. Psychol. Learn. Mem. Cognit. 20, 1322-1340.

34. Logan, G. D. (1988) Psychol. Rev. 95, 492-527.

35. Suppes, P. \& Liang, L. (1998) in Recent Progress in Mathematical Psychology, eds. Dowling, C., Roberts, F. S. \& Theuns, P. (Lawrence Earlbaum Assoc., Hillsdale, NJ).

36. Bisley, J. W. \& Pasternak, T. (2000) Cereb. Cortex 10, 1053-1065.

37. Recanzone, G. H., Merzenich, M. M. \& Schreiner, C. E. (1992) J. Neurophysiol. 67, 1071-1091.

38. Elbert, T., Pantev, C., Wienbruch, C., Rockstroh, B. \& Taub, E. (1995) Science 270, 305-307.

39. Durup, G. \& Fessard, A. (1935) Annu. Psych. 36, 266-281.

40. Bakin, J. S. \& Weinberger, N. M. (1990) Brain Res. 536, 271-286.

41. Weinberger, N. M., Ashe, J. H., Metherate, R., McKenna, T. M., Diamond, D. M. \& Bakin, J. (1990) Concepts Neurosci. 1, 91-131.

42. Recanzone, G. H., Schreiner, C. E. \& Merzenich, M. M. (1993) J. Neurosci. 13, 87-103.

43. Crist, R. E., Li, W. \& Gilbert, C. D. (2001) Nature Neurosci. 4, 519-525.

44. Schoups, A., Vogels, R., Qian, N. \& Orban, G. (2001) Nature 412, 549-553.

45. Ghose, G. M., Yang, T. \& Maunsell, J. H. R. (2002) J. Neurophysiol. 87, 1867-1888.

46. Mollon, J. D. \& Danilova, M. V. (1996) Spatial Vis. 10, 51-58.

47. Deneve, S., Latham, P. E. \& Pouget, A. (2001) Nature Neurosci. 4, 826-831.

48. Hays, W. L. (1988) Statistics (Holt, Rinehart \& Winston, Fort Worth, TX), 4th Ed. 